\title{
Detection of Alternative Drugs for Illegal Injection Based on Surface-Enhanced Raman Spectroscopy
}

\author{
Lin Bao $\mathbb{D},,^{1,2}$ Siqingaowa Han $\mathbb{D},{ }^{3}$ Xuanyu Sha $\mathbb{D},{ }^{1}$ Hang Zhao $\mathbb{D}^{1},{ }^{1}$ Yuping Liu $\mathbb{D}^{1},{ }^{1}$ \\ Dianyang Lin $\mathbb{D}$, ${ }^{1}$ and Wuliji Hasi $\mathbb{D}^{1}$ \\ ${ }^{1}$ National Key Laboratory of Science and Technology on Tunable Laser, Harbin Institute of Technology, Harbin 150080, China \\ ${ }^{2}$ College of Physics and Electronics Information, Inner Mongolia University for Nationalities, Inner Mongolia, \\ Tongliao 028043, China \\ ${ }^{3}$ Affiliated Hospital, Inner Mongolia University for the Nationalities, Inner Mongolia, Tongliao 028007, China
}

Correspondence should be addressed to Siqingaowa Han; hansiqin@126.com, Yuping Liu; 15b321005@hit.edu.cn, and Dianyang Lin; dianyanglin@hit.edu.cn

Received 18 November 2018; Accepted 28 February 2019; Published 1 October 2019

Academic Editor: Nicolae Leopold

Copyright ( 2019 Lin Bao et al. This is an open access article distributed under the Creative Commons Attribution License, which permits unrestricted use, distribution, and reproduction in any medium, provided the original work is properly cited.

\begin{abstract}
Promethazine and scopolamine are two major components of a drug commonly used as a substitute for heroin addiction but with its own potential for abuse. Here, surface-enhanced Raman spectroscopy experiments were combined with density functional theory computations of the Raman frequencies of promethazine. The enhancement of the SERS substrate was optimized by contrast detection of silver nanoparticles. The optimized substrate was used to detect scopolamine, promethazine and their mixtures. Several mixtures of different concentrations were detected and analyzed. The approach of rapid identification and monitoring of drugs will become an important tool in the detection and control of illicit drug use.
\end{abstract}

\section{Introduction}

Heroin is one of the most common opioid substances used by drug addicts. The cognitive impairment of heroin addicts is mainly related to memory function, impulse control, selective attention, processing speed of cognitive transfer information, and so on [1-3]. In recent years, a new type of illegal intravenous drug has emerged in some areas to replace heroin $[4,5]$. The main constituents are scopolamine, promethazine, and buprenorphine, abbreviated BSP [5]. BSP is cheaper than heroin and is relatively easy to obtain. Some people were initially used to detoxify heroin, and then, new drug abuse was developed. So that instead of injecting heroin, BSP addicts, even though they did not use heroin, continued to increase their cognitive impairment $[1-3,6]$. The patients reported that the pleasure was even more intense than that produced by heroin. BSP abuse was more likely to damage cortical neurons than heroin, and the recovery of gray matter in the brain was slower than in heroindependent groups. This suggests that the damage to cortical neurons caused by BSP abuse is difficult to recover in a short time. This new type of drug with potential for abuse and addiction, if not detected in time, is reported and included international control that makes it possible for such drugs to enter a range of international drug trade activities. It is a major challenge and subject for drug control work to establish a long-term detection and investigation mechanism and to find out new drug-related abuse problems in a timely manner. The detection of social hazards caused by abuse and substance abuse is an important part of drug control.

Buprenorphine is currently under strict control and is not readily available [7]. Thus, we analyzed scopolamine, promethazine, and their mixture. Our group has qualitatively and quantitatively analyzed scopolamine [8] and identified its Raman peaks. This laid the foundation for this work. Here, we calculated the theoretical Raman peak of promethazine and compared that to the experimental spectrum. We then optimized the experimental conditions and detected several mixed samples to evaluate this analytical utility of this approach. 
Raman spectroscopy can be used to analyze solids, gases, and liquids, but its signal is weak, which is not conducive to the application of detection, especially for low concentration samples. However, the development of nanotechnology has greatly promoted the development of Raman technology [9]. The sensitivity of Raman spectroscopy combined with surfaceenhanced Raman spectroscopy (SERS) of nanomaterials has increased its sensitivity by at least 7 10 orders of magnitude [10]. Two widely acknowledged enhancement mechanisms are electromagnetic [11] and chemical enhancement [12]. The electromagnetic mechanism mainly contributes to singlemolecule sensitivity in SERS. SERS "hotspots," which are interparticle junctions in silver and gold nanoaggregates, where the local EM-field amplitude increases by plasmonic field confinement, enable the vast enhancement of Raman signal intensity based on the fourth power of the ratios between the local EM fields and external incident fields [11]. The most studied mechanism for the chemical enhancement is the socalled charge-transfer (CT) mechanism. This mechanism may involve the presence of a CT complex either by direct binding to the metal or by indirect binding with the assistance of an electrolyte ion [12]. SERS has been widely applied to identify or detect illicit drugs, including trace illicit drugs, suspicious objects and drugs, or their metabolites in real biological system (urine, saliva, and so on) [13]. Currently, SERS researches about illicit drug detection are still in the development stage. The improved Raman analyzer will provide the possibility to make SERS a practically analytical technique. SERS is hopeful to be a versatile and powerful sensor platform in real-world applications for illicit drug analysis. The experimental workflow is shown in Figure 1.

\section{Experimental}

2.1. Materials and Reagents. Two types of silver nanoparticles were obtained by reduction with sodium citrate and ascorbic acid using silver nitrate as the metal precursor; these were used as the SERS substrate. Next, scopolamine injection (50 mg in $2 \mathrm{~mL}$ ) and promethazine injection $(0.3 \mathrm{mg}$ in $1 \mathrm{~mL})$ were serially diluted. The Raman spectra of scopolamine powder and promethazine powder were collected as the standard reference. Ultrapure water $(18 \mathrm{M} \Omega \cdot \mathrm{cm})$ was used as the solvent for dilution and solution allocation throughout the experiment.

2.2. Apparatus. The heating agitator was used to prepare the silver sol. The liquid moving gun extracts the sample droplets and is placed on the detection platform. A portable Raman spectrometer collected the Raman spectra. A scanning electron microscope (SEM) was used to characterize the morphology and size of the nanoparticles. A vortex oscillator was used to mix the solutions quickly and evenly.

This experiment used a BWS415-785H (B\&W Tek, Inc.) portable Raman spectrometer with $785 \mathrm{~nm}$ laser excitation, spectral resolution less than $3 \mathrm{~cm}^{-1}$, with a wavelength range of $68-2700 \mathrm{~cm}^{-1}$. The data acquisition, processing, and background subtraction are all done with Bwram1.01.20 software. The focal length of the focused beam is $6.8 \mathrm{~mm}$.
The test power is $90 \mathrm{~mW}$ with 5-s acquisition times. The laser beam diameter is $10 \mu \mathrm{m}$.

2.3. Preparation of Silver Sol. We used the synthesis described by Lee and Meisel [14]. Here, $0.045 \mathrm{~g}$ silver nitrate was added to a clean beaker and dissolved with deionized water to $0.18 \mathrm{~g} / \mathrm{L}$ in a $250-\mathrm{mL}$ flask. This was heated with magnetic stirring and treated with $0.114 \mathrm{~g}$ sodium limonate in $10 \mathrm{~mL}$ water and $1 \%$ citric acid by mass. After boiling, $5 \mathrm{~mL}$ sodium citrate was added quickly while holding the reaction temperature at $100^{\circ} \mathrm{C}$ with stirring. The solution gradually changed from colorless to golden over one hour. The solution eventually became grayish green. We then removed the heat and cooled the sample to room temperature and stored the resulting silver nanoparticles in a brown bottle at $4^{\circ} \mathrm{C}$ without light. After the silver sol was prepared, it was washed with water several times via centrifugation.

An alternative method is from Qin et al. [15]. Ascorbic acid $\left(6.0 \times 10^{-4} \mathrm{~mol} / \mathrm{L}\right)$ was used to reduce silver nitrate $(2.5 \mathrm{~mL} 0.1 \mathrm{~mol} / \mathrm{L})$. Sodium citrate was used as a stabilizer. A mixture of ascorbic acid and sodium citrate was heated to $30^{\circ} \mathrm{C}$ in a water bath and then quickly treated with silver nitrate. It was stirred at $900 \mathrm{r} / \mathrm{min}$ for 15 minutes and then heated to $100^{\circ} \mathrm{C}$ for 2 hours. After natural cooling to room temperature, it was stored at $4^{\circ} \mathrm{C}$ in the dark.

\section{Results and Discussion}

Quantum chemical calculation of promethazine was performed using the density functional theory. The B3LYP hybrid function combined with the $6-31 \mathrm{~g}(\mathrm{~d}, \mathrm{p})$ basis set was used to optimize the molecular structure, and the theoretical Raman spectra were calculated. The optimized structure of promethazine is shown in Figure 2. The Raman spectra of promethazine solid powder and SERS of the aqueous solution were collected and compared with the calculated theoretical Raman spectra of promethazine (Figure 3).

We used the Raman spectrum of the powder as the standard reference. If the theoretical calculation is not different from its comparison, then it is considered to be successful. The peak assignment of the theoretical characteristic peak compares the SERS spectrum to the normal Raman spectrum. If there is a corresponding peak position in the SERS spectrum and the peak intensity obviously increases, then it can be used as a basis for qualitative or quantitative sample analysis. Figure 3 shows theoretical calculations of promethazine. The Raman and SERS peaks of the solid powders are shown in Table 1. In general, the SERS lines at most Raman peaks are slightly offset from the theoretical calculations - this might be attributed to the adsorption of the sample molecules on the surface of the silver nanoparticles and their interactions during SERS detection. This results in a shift in the Raman spectrum [16]. The theoretical peak calculations in a vacuum and the actual environment are inconsistent with the literature [17]. The peaks of scopolamine have been described previously [8].

Now that the characteristic peaks of the two samples have been determined, we next compared the two sols under 


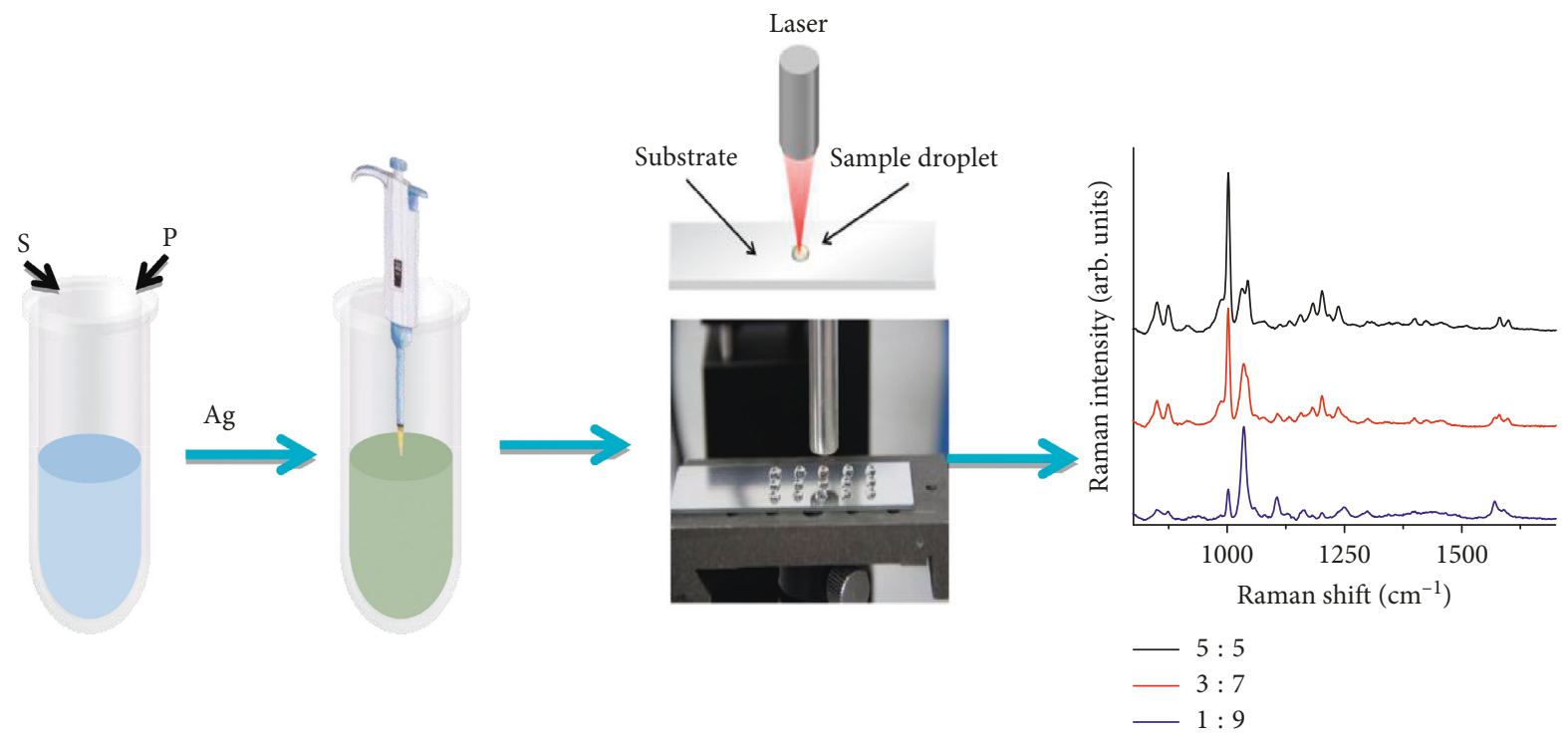

FIgURE 1: Schematic diagram of main process of experiment. S: scopolamine; P: promethazine.

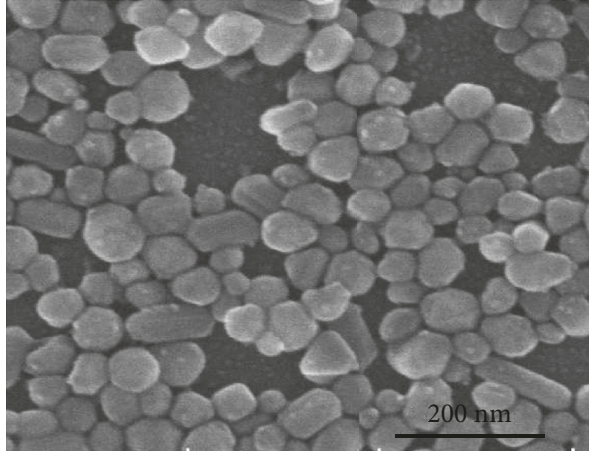

(a)

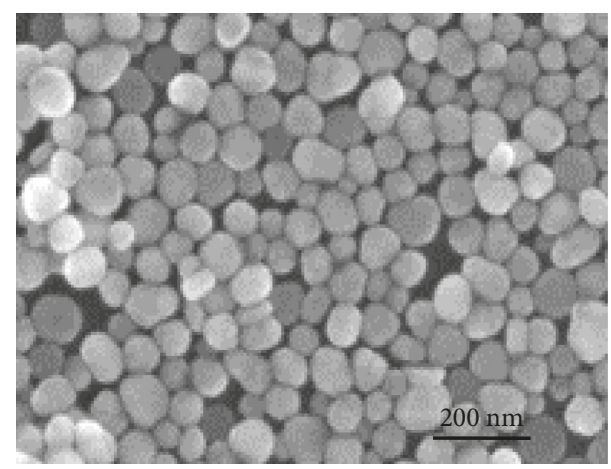

(b)

FIgURE 2: SEM characterization of two silver sols.

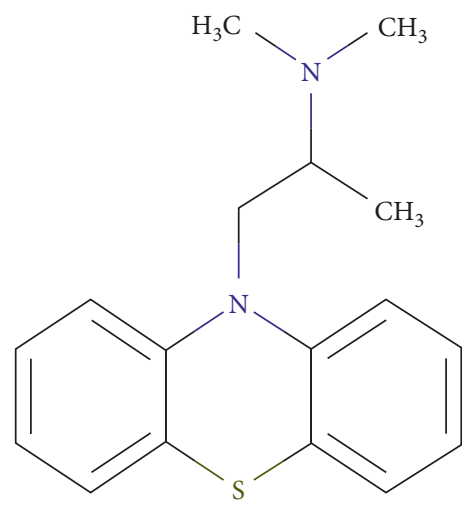

(a)

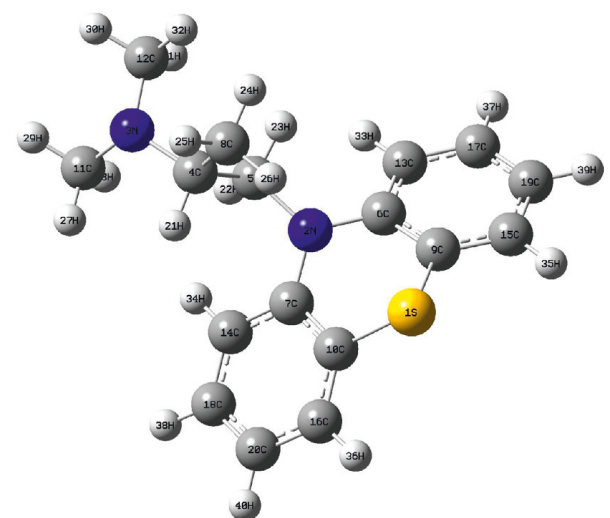

(b)

FIGURE 3: Molecular structure of promethazine.

the same conditions. We prepared two kinds of silver sols according to Lee and Meisel [14] and Qin et al. [15] (Figures 4(a) and 4(b)). Figure 4 compares the SEM of two silver sol nanoparticles. Figure 4(a) shows that the yield is low with many rod-shaped particles. The spherical particles are irregular and uneven. These are worse than the nanoparticles shown in Figure 4(b), which are monodisperse and stable. 
TABLE 1: Assignment of the Raman characteristic peaks of promethazine.

\begin{tabular}{lccc}
\hline $\begin{array}{l}\text { Solid Raman wave number } \\
\left(\mathrm{cm}^{-1}\right)\end{array}$ & $\begin{array}{c}\text { SERS wave number } \\
\left(\mathrm{cm}^{-1}\right)\end{array}$ & $\begin{array}{c}\text { Calculated wave number } \\
\left(\mathrm{cm}^{-1}\right)\end{array}$ & Assignment \\
\hline 1034 & 1034 & 1030 & Double benzene ring internal symmetric bending \\
1107 & 1112 & 1085 & C-S-C stretching + benzene ring bending \\
C-N-C stretching & Benzene ring internal symmetric \\
1292 & 1254 & 1236 & $\begin{array}{c}\text { stretching } \\
\text { stroctic ring symmetric }\end{array}$ \\
1571 & & 1306 & Benzene ring internal symmetric stretching \\
\hline
\end{tabular}

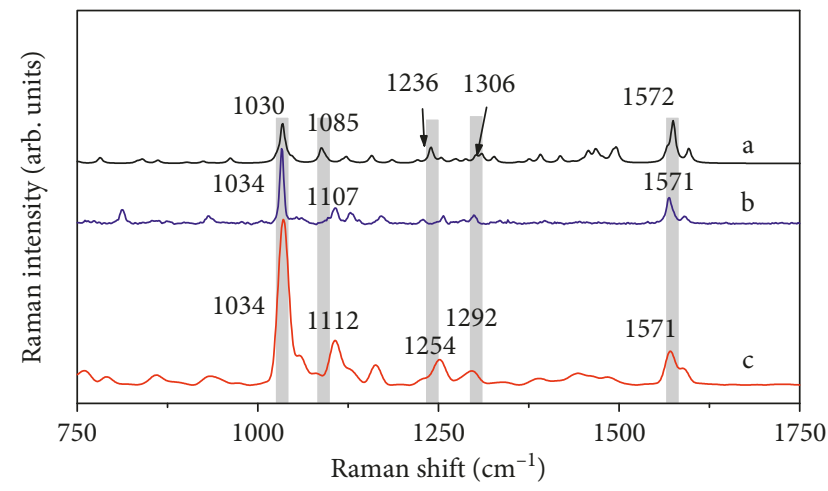

FIgUre 4: Promethazine: (a) theoretical Raman spectra, (b) solid Raman spectra, and (c) SERS spectra.

Then, we studied five replicates for each group (Figure 5). A represents the silver sol in Figure 2(b), and B represents the first silver sol in Figure 2(a). The most prominent Raman peaks of promethazine are $1034 \mathrm{~cm}^{-1}$, $1112 \mathrm{~cm}^{-1}$, and $1571 \mathrm{~cm}^{-1}$ as shown in Figure 5(a). It can be observed that the peak intensity of the characteristic peak detected by sol A [15] is higher than that of sol B [14]. Figure 5(b) shows that the enhancement effects of both sols are equal for scopolamine. After comprehensive analysis, Figure 4(b) was selected as the ideal substrate.

The initial concentration of scopolamine and promethazine is $5^{-4} \mathrm{M}$. The volume ratio of concentrated silver sol to the sample is $1: 1$, and the initial concentration ratio of scopolamine to promethazine is $5: 5$. The volume ratio of scopolamine to promethazine is $5: 5,3: 7$, and $1: 9$ (Figure 6). The specific proportion of promethazine was higher than that of scopolamine although the proportion of promethazine varied according to the unique dose. At ratio of $5: 5$, and the $1034 \mathrm{~cm}^{-1}$ peak of promethazine overlapped with the $1032 \mathrm{~cm}^{-1}$ peak of scopolamine. When the promethazine concentration ratio increased to $3: 7$, there was a single peak of promethazine at $1034 \mathrm{~cm}^{-1}$ and an obvious characteristic peak of promethazine with no $1044 \mathrm{~cm}^{-1}$ scopolamine peak. When the mixing ratio was 1 : 9 , the single peak at $1034 \mathrm{~cm}^{-1}$ of promethazine was obvious as was the peak at $1112 \mathrm{~cm}^{-1}$; the $1002 \mathrm{~cm}^{-1}$ peak of scopolamine decreased obviously however. The characteristic peak intensity is proportional to the doping ratio. At $1: 9$, their main characteristic peaks can be observed directly in the Raman spectra-this agrees with the actual doping ratio, which lays the foundation for rapid characterization of the mixed samples. Due to the competitive

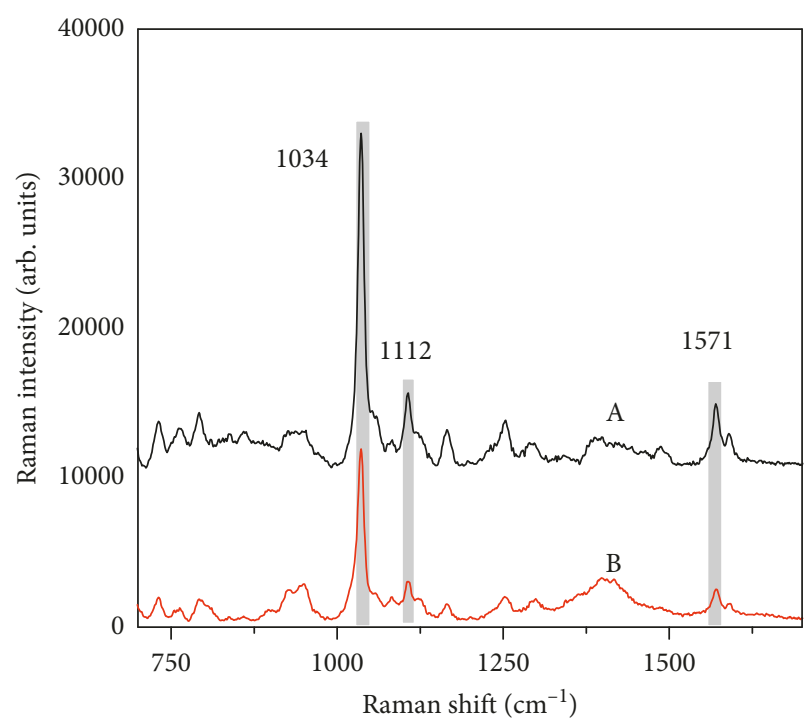

(a)

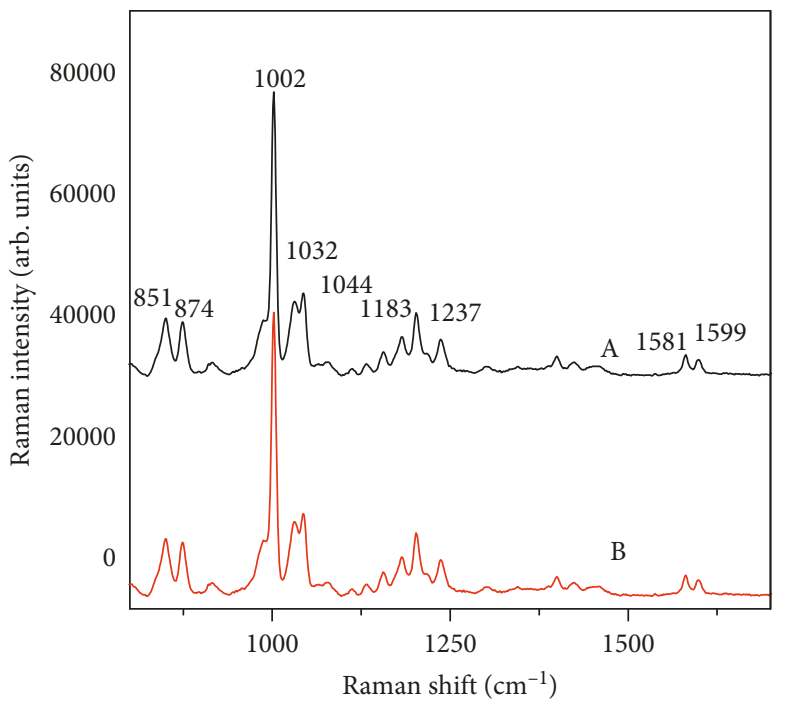

(b)

FIgURE 5: Comparison of the SERS effects of two silver sols with (a) promethazine and (b) scopolamine. A represents the second silver sol in Figure 2 and B represents the first silver sol in Figure 2; both at $5^{-4} \mathrm{M}$.

adsorption of the mixed samples on the substrate, the mixing ratio on the substrate is different from that in solution; thus, quantitative analysis of the SERS mixture is complicated and will be studied in the future. 


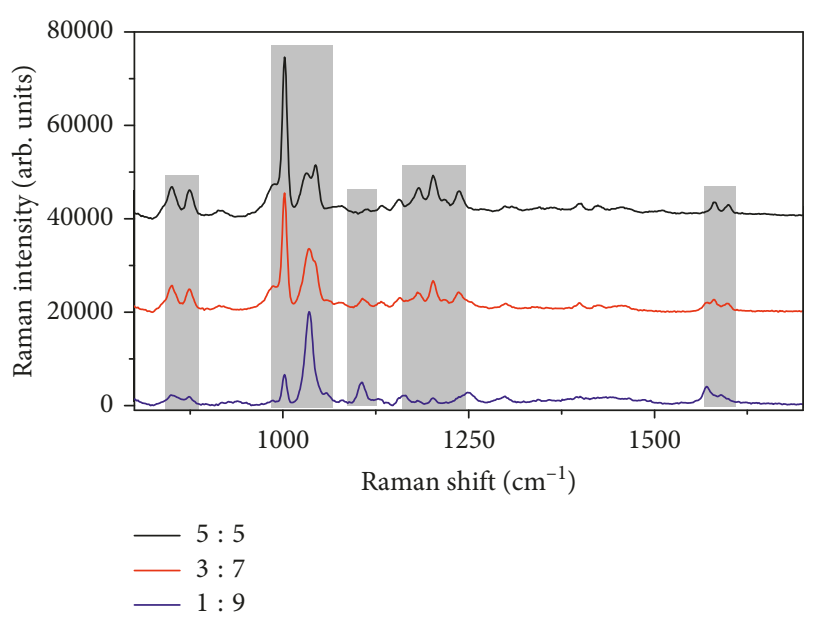

FIGURE 6: SERS spectra with different mixing ratios.

\section{Conclusions}

The theoretical Raman spectra of promethazine were obtained, and the characteristic peaks were determined. The SERS spectra at different mixing ratios were determined and combined with characteristic Raman peak assignments from scopolamine. The spectrogram suggests that both of them exist concurrently. These data show that qualitative analysis can be used to unmix a complex Raman spectrum. This approach is an effective analytical tool for the detection and monitoring of drugs of abuse.

\section{Data Availability}

All the data included in this study are available upon request by contact with the corresponding author.

\section{Conflicts of Interest}

The authors declare that they have no financial and personal relationships with other people or organizations that can inappropriately influence their work.

\section{Acknowledgments}

This work was supported by the National Natural Science Foundation of China (Grant no. 31871873), Inner Mongolia University for the Nationalities (Grant no. NMDYB18006), and the Inner Mongolia Autonomous Region Natural Science Foundation of China (Grant no. 2018LH08055).

\section{References}

[1] T. M. C. Lee, W.-H. Zhou, X.-J. Luo, K. S. L. Yuen, X.-Z. Ruan, and X.-C. Weng, "Neural activity associated with cognitive regulation in heroin users: a fMRI study," Neuroscience Letters, vol. 382, no. 3, pp. 211-216, 2005.

[2] T. Lundquist, "Cognitive consequences of cannabis use: comparison with abuse of stimulants and heroin with regard to attention, memory and executive functions," Pharmacology Biochemistry and Behavior, vol. 81, no. 2, pp. 319-330, 2005.

[3] T. Ornstein, J. L. Iddon, A. M. Baldacchino et al., "Profiles of cognitive dysfunction in chronic amphetamine and heroin abusers," Neuropsychopharmacology, vol. 23, no. 2, pp. 113126, 2000.

[4] Z. Denghui, Z. Dengke, D. Huiqiong et al., "Effects of buprenorphine sublingual tablets combined with scopolamine and promethazine on cognitive function," Chinese Journal of Drug Dependence, vol. 15, no. 2, pp. 35-39, 2006.

[5] Z. Xuhui, W. Xuyi, L. Jun et al., "A study of brain gray matter density in patients with buprenorphine sublingual tablets combined with scopolamine and promethazine," Chinese Journal of Clinical Psychology, vol. 19, no. 3, pp. 285-288, 2011.

[6] B. Devan, D. Sierramercadojr, M. Jimenez et al., "Phosphodiesterase inhibition by sildenafil citrate attenuates the learning impairment induced by blockade of cholinergic muscarinic receptors in rats," Pharmacology Biochemistry and Behavior, vol. 79, no. 4, pp. 691-699, 2004.

[7] Z. Dengke, Z. Xuhui, and Z. Xuehui, "Investigation of mixed abuse of buprenorphine, scopolamine and promethazine in Changsha," Chinese Journal of Drug Dependence, vol. 15, no. 1, pp. 53-57, 2006.

[8] B. Lin, H. Siingaowa, S. Xuanyu et al., "Detection of scopolamine hydrobromide via Surface-enhanced Raman Spectroscopy (SERS)," Analytical Sciences, vol. 33, no. 11, pp. 1237-1241, 2017.

[9] Y.-J. Ai, P. Liang, Y.-X. Wu et al., "Rapid qualitative and quantitative determination of food colorants by both Raman spectra and Surface-enhanced Raman Scattering (SERS)," Food Chemistry, vol. 241, pp. 427-433, 2018.

[10] M. Rycenga, C. M. Cobley, J. Zeng et al., "Controlling the synthesis and assembly of silver nanostructures for plasmonic applications," Chemical Reviews, vol. 111, no. 6, pp. 36693712, 2011.

[11] Y. S. Yamamoto and T. Itoh, "Why and how do the shapes of surface-enhanced Raman scattering spectra change? Recent progress from mechanistic studies," Journal of Raman Spectroscopy, vol. 47, no. 1, pp. 78-88, 2016.

[12] Y. Liu, Z. Lu, H. Zhu, and W. Hasi, "Characterization of a chloride-activated surface complex and correspongding enhancemengt mechanism by SERS saturation effect," Journal of Physical Chemistry C, vol. 121, no. 1, pp. 950-957, 2017.

[13] B. Yu, M. Ge, P. Li, Q. Xie, and L. Yang, "Development of surface-enhanced Raman spectroscopy application for determination of illicit drugs: towards a practical sensor," Talanta, vol. 191, pp. 1-10, 2019.

[14] P. C. Lee and D. Meisel, "Adsorption and surface-enhanced Raman of dyes on silver and gold sols," Journal of Physical Chemistry, vol. 86, no. 17, pp. 3391-3395, 1982.

[15] Y. Qin, X. Ji, J. Jing, H. Liu, H. Wu, and W. Yang, "Size control over spherical silver nanoparticles by ascorbic acid reduction," Colloids and Surfaces A: Physicochemical and Engineering Aspects, vol. 372, no. 1-3, pp. 172-176, 2010.

[16] L. Liu, N.-J. Kim, H. Li, Z. Hu, and M. Lin, "Use of a fractallike gold nanostructure in surface-enhanced Raman spectroscopy for detection of selected food contaminants," Journal of Agricultural and Food Chemistry, vol. 56, no. 21, pp. 9843-9847, 2008.

[17] M. Prabhaharan, A. R. Prabakaran, S. Gunasekaran, and S. Srinivasan, "Molecular structure and vibrational spectroscopic investigation of melamine using DFT theory calculations," Spectrochimica Acta Part A: Molecular and Biomolecular Spectroscopy, vol. 123, pp. 392-401, 2014. 

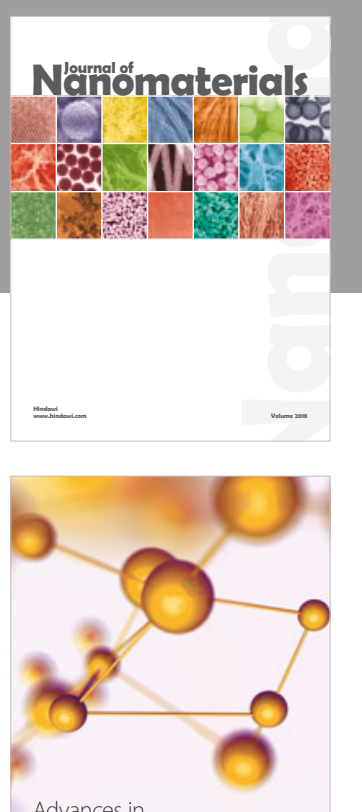

Physical Chemistry
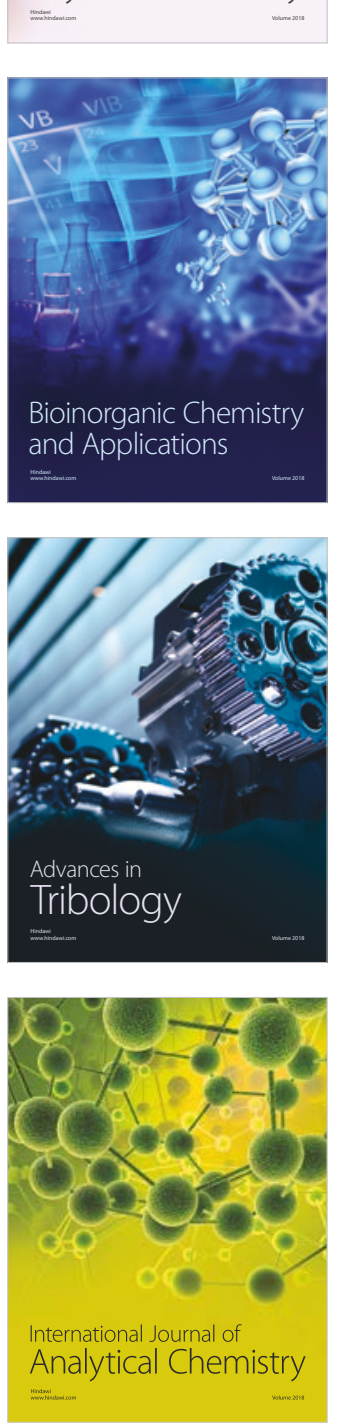

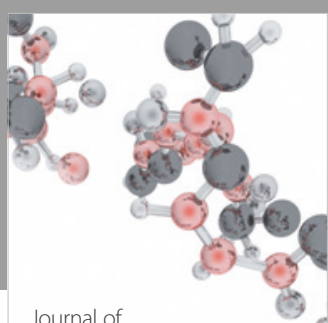

Analytical Methods

in Chemistry

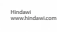

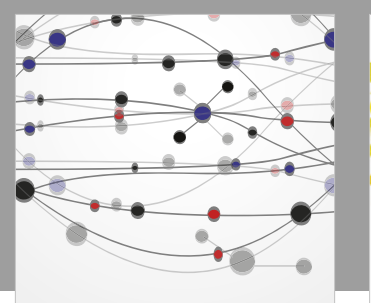

The Scientific World Journal

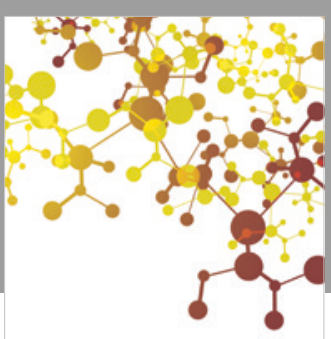

Journal of

Applied Chemistry
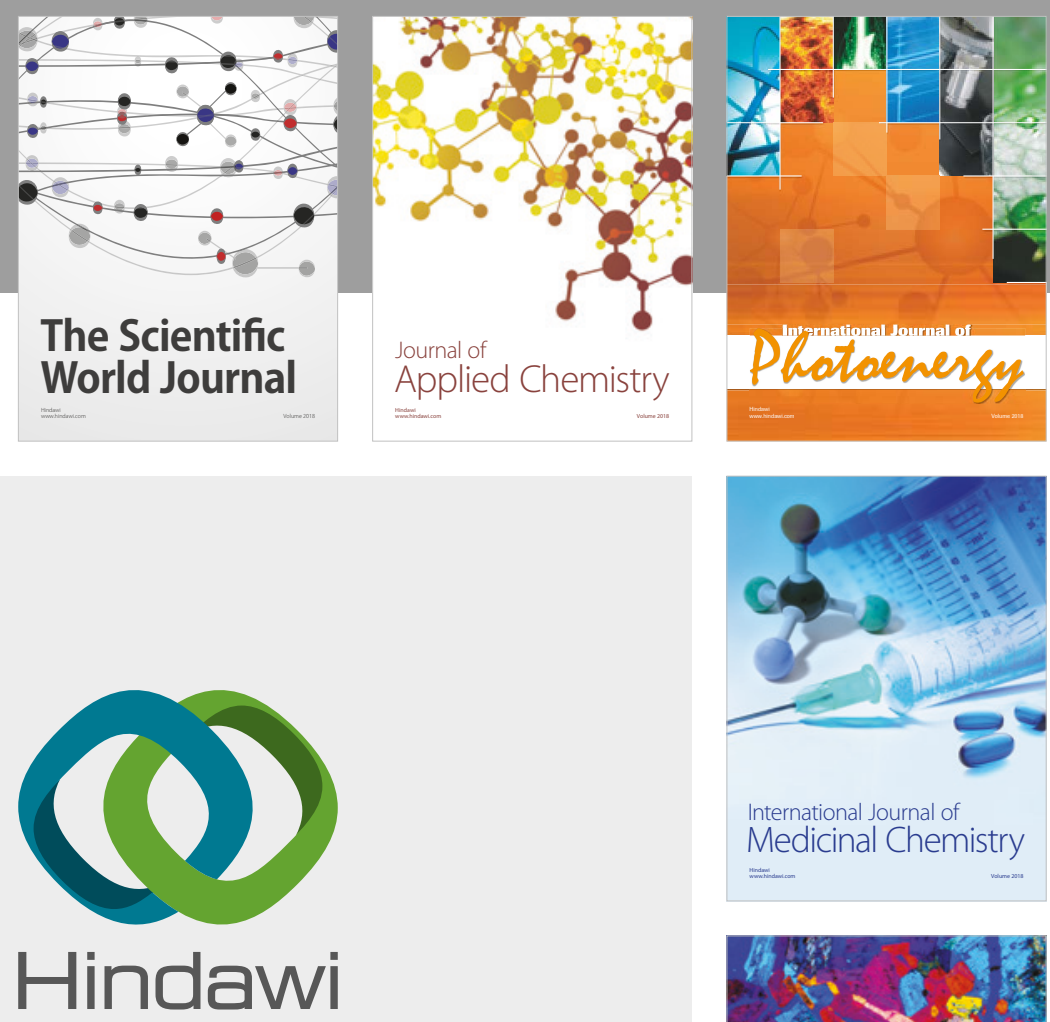

Submit your manuscripts at

www.hindawi.com
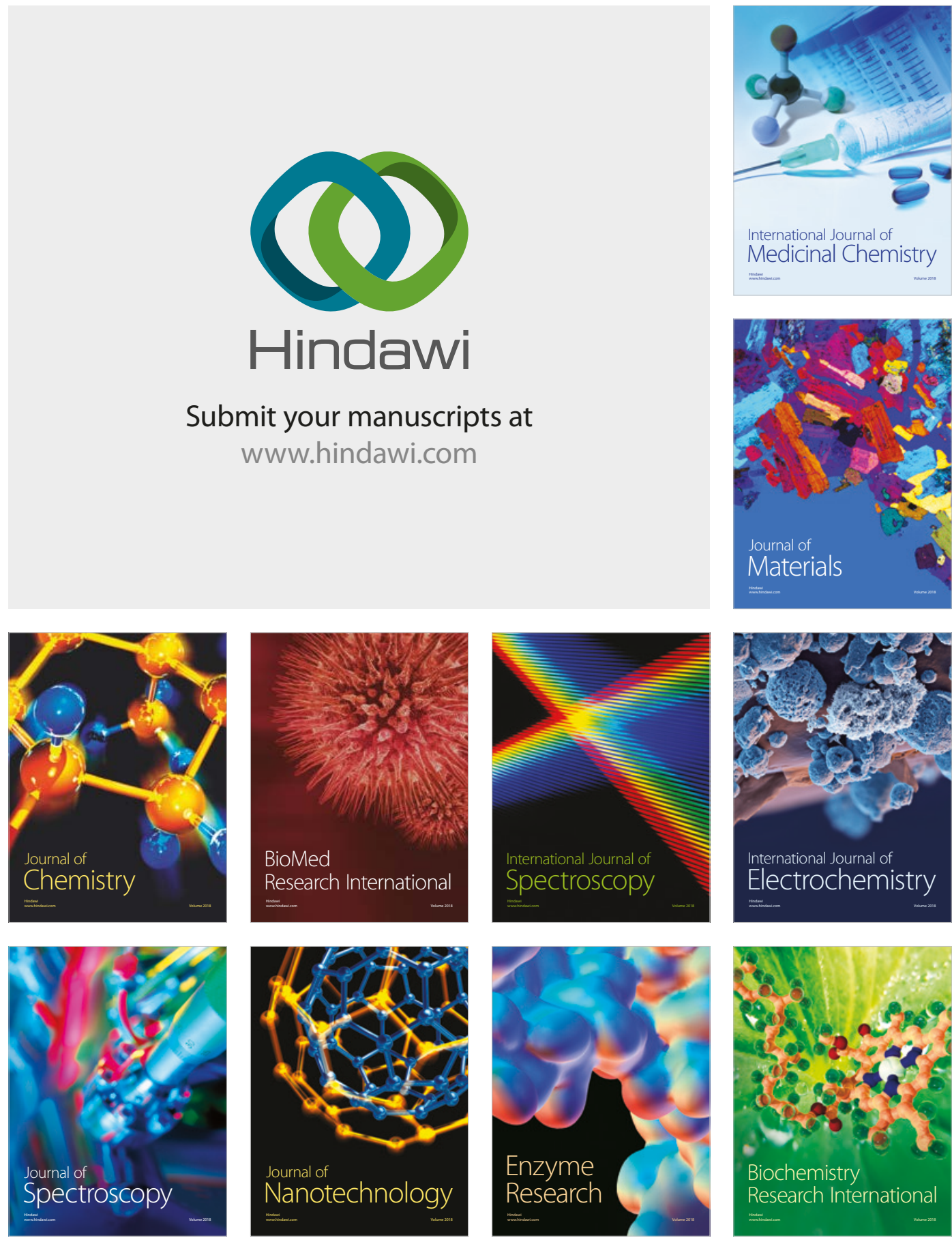
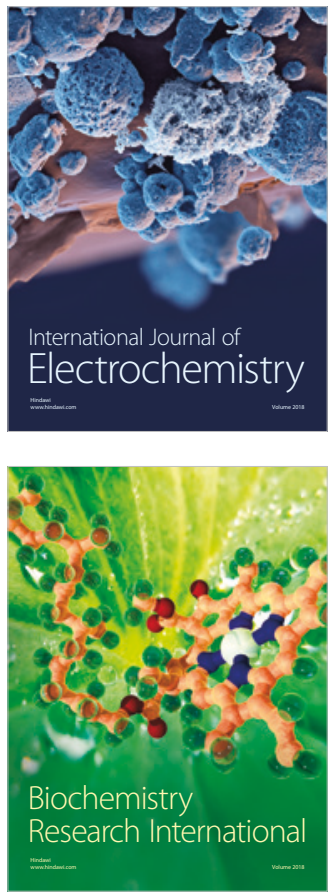